\title{
NEUROCYSTICERCOSIS IN CHILDREN AT GMC, CHARAK HOSPITAL, POKHARA
}

\author{
SP Shrestha ${ }^{1 *}$, S Dhungana ${ }^{2}$ and AK Shrestha ${ }^{3}$ \\ Department of Pediatrics, Chitwan Medical College, Bharatpur-10, Chitwan, Nepal. \\ 2 Department of Radiology, Chitwan Medical College, Bharatpur-10, Chitwan, Nepal. \\ ${ }^{3}$ Department of Community Medicine, Chitwan Medical College, Bharatpur-10, Chitwan, Nepal. \\ *Correspondence to : Dr SP Shrestha, Department of Pediatrics, Chitwan Medical College, Bharatpur-10, Chitwan, Nepal. \\ E-mail:dr_shrestha_shivanita@yahoo.com
}

\begin{abstract}
Neurocysticercosis (NCC) is a common cause of seizure and major cause of neurological illness globally. It is one of the major causes of epilepsy in children. Fewer studies were done in the past regarding NCC in Nepal. However study on NCC in children in Gandaki Medical College (GMC), Charak Hospital is the first of its kind. 23 children with CT scanning of head that were consistent with NCC over a period of 18 months at GMC, Charak Hospital were included as study subjects. Although there are so many diagnostic tools including MRI, immunological and serological tests for the detection of NCC, CT scanning of the head was used for its diagnosis in this study. $17.3 \%$ of the study subjects were found to have suffered from NCC. Girls $(60 \%)$ outnumbered boys $(40 \%)$; it was more commonly seen in $10-15$ years age group $(47.83 \%)$. Three main clinical presentations were Seizure (100\%), Headache (73.91\%) and Vomiting (30.43\%). Most of the cases had ring enhancing lesion at colloidal stage. All patients were treated with albendazole, dexamethasone and antiepileptic drugs (AED) and advised strongly to continue for the period of at least 2 years without failure. NCC should be considered and CT/MRI head must be performed in all children above 5 years of age presented as seizure, headache or vomiting where Taenia solium (TS) infestations are found endemic.
\end{abstract}

Key Words: Neurocysticercosis, Epilepsy \& Seizure.

\section{INTRODUCTION}

Neurocysticercosis (NCC) is a most common parasitic infection of CNS affecting approximately 50 million people worldwide ${ }^{1}$ and has become a major cause of neurological disease too. ${ }^{3}$ Three quarters of the 50 million people with epilepsy live in the poor countries of the world and $94 \%$ are untreated. ${ }^{2}$ WHO has estimated that more than 50,000 people die of NCC alone each year. ${ }^{1}$ This disease is becoming increasingly more common especially in India, China, Indonesia, South and Central America. It has been estimated that 20-50 per cent of epilepsy may be due to neurocysticercosis in this region. ${ }^{4}$

NCC is common in the communities with poor hygiene and sanitation where human faeces are used in the field/ garden as fertilizer. NCC can occur both in vegetarian and non -vegetarians. Vegetarians might be infected by eating raw vegetables contaminated with ova or proglottids of Taenia solium (TS). Open field defecation, unfortunately a common practice in rural and sub urban areas is a potent source of soil and vegetable contamination resulting NCC. ${ }^{5}$ Non vegetarians get infected after eating improperly cooked pork meat that contains cysticerci cellulose. Therefore, man develops NCC by three possible mechanisms; 1) by drinking water or food contaminated by ova or proglottids of TS. 2) Auto infection due to improper hand hygiene after defecation and 3) by regurgitation of ova/ proglottids from the small intestine into the stomach. ${ }^{5}$ In all these three modes of infection, the infective stage is ova or eggs of TS. The ingested or regurgitated ova undergo enzymatic digestion of their wall in the stomach or upper small intestine and liberate the oncospheres which penetrate through intestinal epithelium to reach the lymphatics or blood vessels and thus can spread to any site.

Clinical manifestation of NCC depends on the site involved. Neurological symptoms arise as a result of host's inflammatory response to dying parasite. ${ }^{6,10}$ It may affect brain parenchyma, intra-ventricular system, spinal cord, sub-arachnoids space and eyes. Seizure develops if there is brain parenchyma involvement.

\section{LIFE CYCLE}

Taenia solium (TS) is also known as 'armed tape worm' of man. It infects human by eating raw or insufficiently cooked pork meat that contains cysticerci. The adult TS are 2-3 meter in length and lives in the upper jejunum. Scolex is $1 \mathrm{~mm}$ in diameter, almost a size of pin head. It bears 4 suckers and is globular outlined. Head contains rostellium with doubled row of alternating large and small hooklets. Neck is short with 5-10 
$\mathrm{mm}$ in length. It has proglottids of $800-1000$ in number. TS can survive as long as 25 years. ${ }^{7}$

Adult worm habitat in man and is a definitive host. Adult worm passes ova/ proglottids in the faeces. Pigs that are grazing in the field swallow ova/ proglottids present in the human faeces.
Once ova reach in the intestine of the pig, outer coat is ruptured and oncospheres are liberated. These oncosheres through their hooklets penetrates the intestinal wall and through portal veins and mesenteric lymphatic, they finally reach the systemic circulation. These oncospheres are finally filtered from the systemic circulation and they settle down in different muscles

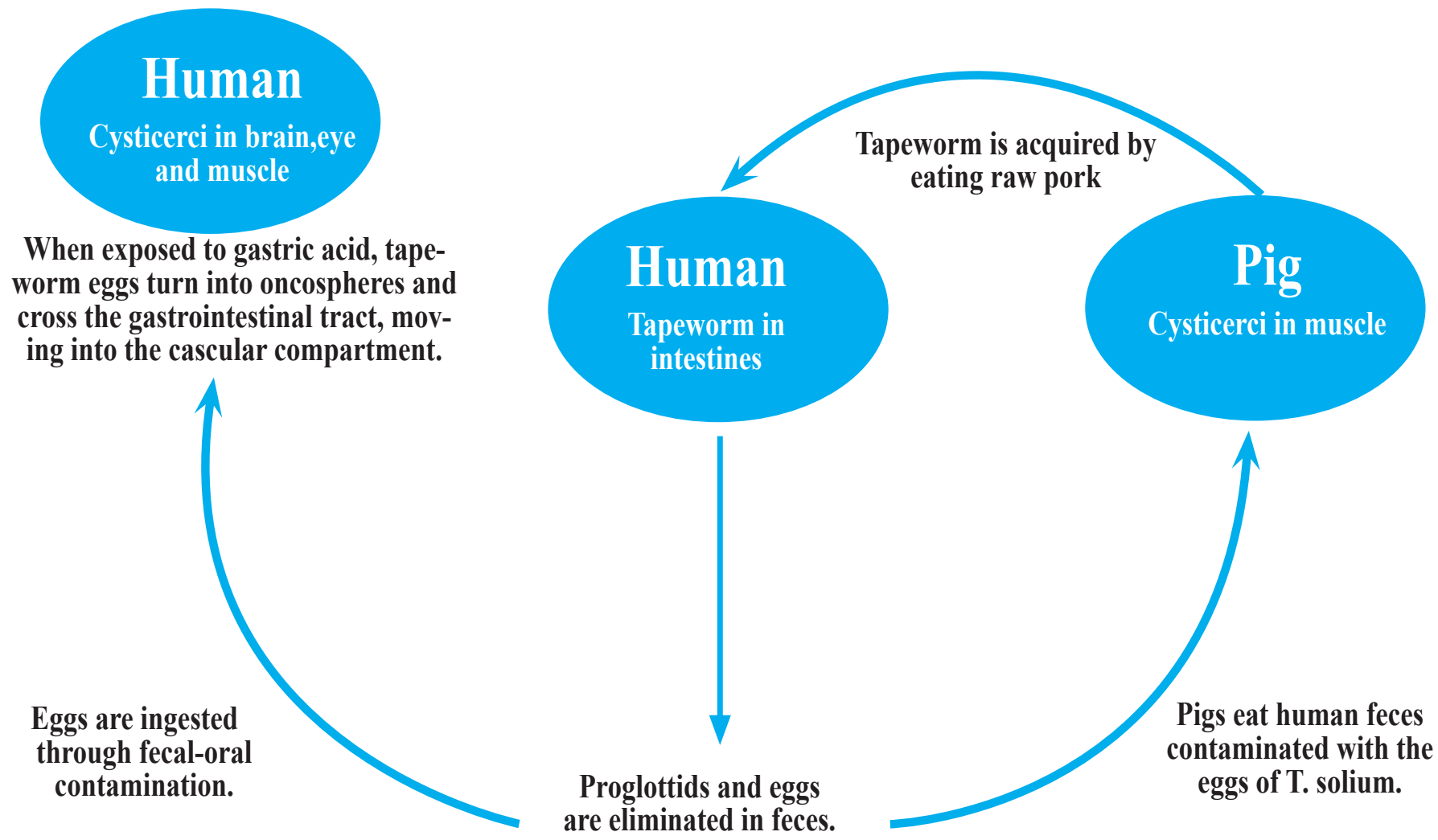

The T. solium cycle is incomplete. The cycle does not progress past the larvae stage and results in cysticerci lodging in human tissue.

(tongue, shoulder, cardiac, neck) where they undergo further development.

Once the oncospheres reach the muscles they loose their hooklets, they undergo central liquefaction in about 8 days after infection. Each oncospheres form oval vesicle which gradually increase in size and becomes "larvae" known as cysticerci cellulose.

Once cysticerci reach in the intestine of man while they consume pork meat, the Scolex anchors to the gut wall by means of suckers and develop into adult tape worm. This adult tape worm gets matured in 2-3 months time and start liberating eggs which are passed by human upon defecation. Then the cycle repeats again.

\section{MATERIAL AND METHODS}

This is a hospital based retrospective study conducted to see the prevalence of NCC in children in Gandaki Medical College
The T. solium cycle is complete- progressing from embryo to larvae to worm to embryo. The pig is the intermediate host.

(GMC), Charak Hospital, Pokhara. A total of 133 children between the ages of 2 to 15 years who have undergone CT scan head because of various clinical presentations at Charak hospital were included in this study. Although most useful diagnostic study for NCC is MRI head 4. We took diagnostic tool as CT head as MRI facility does not exist in this centre at present. Review of medical records was done in all patients who had undergone CT scan head during the period of 18 months (Baisakha 2067 to end of Kartik 2068). Children referred for CT scan of head from other centers and patients without complete medical records were excluded from this study.

Out of 133 CT scan performed in this hospital, CT scan Head of 23 children found to be consistent with NCC comprising $17.3 \%$. Radiographic features with single or multiple focal parenchymal, meningeal, intra-ventricular, sub arachnoids and spinal lesions that were non contrast or contrast enhancing were included in the study. All four stages; Vesicular stage, colloidal stage, granular nodular stage and nodular calcified stages were 
present in the affected children; majority being colloidal stage. No serological test (ELTB) or other antibody detection tests were done in these children due to the unavailability of such facilities in our hospital at present.

Clinical details of each CT proven NCC patient were reviewed. All patients with proven CT scan Head for NCC had seizure at presentation. Headaches and vomiting were second and third most common clinical symptoms. All children had received Anti-epileptic drugs (AED) either Carbamazepin, Phenytoin or Sodium Valproate. Albendazole and Dexamethasone were also given. Dexamethasone was prescribed for first 2-3 days prior to albendazole therapy. Patients were asked to come for follow up in the OPD regularly for dose adjustment and whenever required. It is believed that treatment with anti parasitic drugs along with dexamethasone enhances partial or complete resolution of lesion and reduces the risk of recurrent seizures in children. ${ }^{4}$ Albendazole has been prescribed for the period of 28 days. Patient parents were strongly advised to continue AED for at least 2 years daily without failure. All patients were well informed about the side effects of the AED drugs and they were also asked when to return to the hospital immediately if such side effects were seen. Long period follow up were not done owing to many difficulties.

\section{RESULTS}

Of the total 133 patients, 23 children (17.3\%) had Neurocysticercosis. Normal CT scan was reported in 87 children comprising $(65.4 \%)$ of total study. CT report with other problems were $17.3 \%$

\section{Frequency of Disease}

\begin{tabular}{|l|l|l|}
\hline Diagnosis & Frequency & Percentage \\
\hline Normal CT Scan & 87 & 65.4 \\
\hline Neurocysticercosis & 23 & 17.3 \\
\hline Others & 23 & 17.3 \\
\hline Total & 133 & 100.0 \\
\hline
\end{tabular}

Age wise distribution: Age of the patients ranges from the age of 2 to 15 years. The peak incidence of NCC is seen in the age group of $10-15$ years comprising $47.8 \%$ and is consistent with the study done at Lumbini Zonal hospital by Shrestha BM et.al Distribution of patients by Age group

\begin{tabular}{|l|l|l|}
\hline Age & Number & Percentage \\
\hline $3-5$ yrs & 2 & 8.70 \\
\hline $5-10$ yrs & 10 & 43.48 \\
\hline $10-15$ yrs & 11 & 47.83 \\
\hline Total & 23 & 100.00 \\
\hline
\end{tabular}

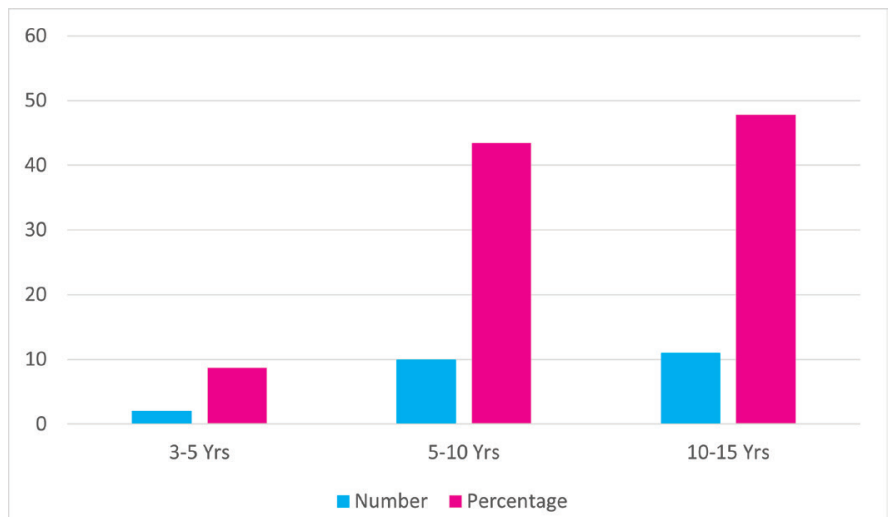

Sex wise distribution of NCC children: Female patient outnumbered male with $60 \%$ female and $40 \%$ male children were affected.

\section{Distribution of patients by Sex}

\begin{tabular}{|l|l|l|l|}
\hline Impression & Male (\%) & Female (\%) & Total \\
\hline Neurocysticercosis & $9(40)$ & $14(60)$ & 23 \\
\hline
\end{tabular}

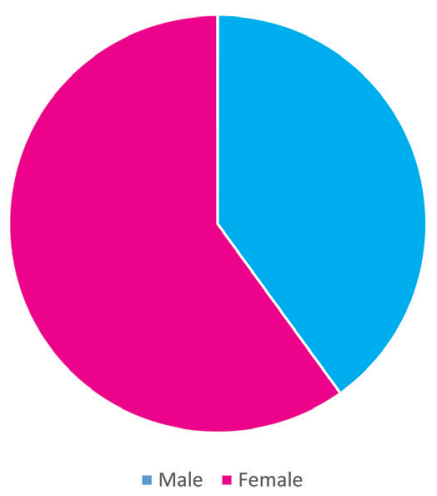

Caste/ Ethnicity: The caste and ethnic distribution of patents were also categorized in this study. The majority of children were from Brahmin background which comprised 30.45 per cent followed by Chhetries comprising 26.09 percent. The number of children from both the Scheduled caste and other caste groups like Gurung, Tamang and Newars constitute 21.74 percent each.

\section{Distribution of patients by caste /ethnicity}

\begin{tabular}{|l|l|l|}
\hline Caste/ Ethnic group & Number & Percentage \\
\hline Brahman & 7 & 30.43 \\
\hline Chhetry & 6 & 26.09 \\
\hline Schedule Caste & 5 & 21.74 \\
\hline Others & 5 & 21.74 \\
\hline Total & 23 & 100 \\
\hline
\end{tabular}


Clinical presentation: most common clinical symptoms at presentation are seizure $100 \%$ of cases followed by headache $73.9 \%$ and then vomiting in $30.43 \%$ among NCC patients

Patients presented with Seizure and additional symptoms $(\mathrm{n}=\mathbf{2 3})$

\begin{tabular}{|l|c|c|}
\hline Symptoms & No & Percentage \\
\hline Fever & 4 & 17.39 \\
\hline Generalized weakness of the body & 4 & 17.39 \\
\hline Vomiting & 7 & 30.43 \\
\hline Up rolling eyes & 6 & 26.08 \\
\hline Stiffening of the body & 4 & 17.39 \\
\hline Headache & 17 & 73.91 \\
\hline Nausea & 1 & 4.34 \\
\hline Loss of consciousness (LOC) & 3 & 13 \\
\hline Abnormal behavior & 1 & 4.34 \\
\hline
\end{tabular}

\section{DISCUSSION}

NCC is the commonest cause of acquired seizure in children. ${ }^{3}$ It is a disease of poverty and under development. ${ }^{2}$

$100 \%$ of children in this study presented with seizure and was the cause of CT scan head done in these children. Most of the seizures are generalized in nature. This finding is not consistent with the study done by Singhi et al, Klara et al ${ }^{8,9}$ and study conducted by BM Shrestha ${ }^{10}$ at Lumbini zonal hospital.

This study showed there were more girls suffering from NCC as compared to boys ( $60 \%$ vs $40 \%$ ). This finding is similar to those studies carried out by Shrestha BM at Lumbini zonal hospital, Ruiz-Garcia, et.al, Thakur, et.al. But this study is in contrast with the finding of Morales et al, where male were predominant. ${ }^{13}$

Current study showed that majority of patients were school going children between the ages of 10-15 years comprising $47.83 \%$ followed by the age group of $5-10$ years. In majority of cases NCC are mostly seen after the age of seven. There were no children below 2 years. These findings are similar to the study done by Kossoff and Thakur et al. ${ }^{6,12}$ Lower prevalence of NCC in toddlers is probably due to prolonged incubation period of TS and might have contributed to the dietary habits in those children where they are little exposed with food from outside as well as contamination to some extent. ${ }^{10}$

Headache and vomiting, the features of raised ICP were present in $73.9 \%$ and $30.4 \%$ of cases respectively. This presentation is almost similar to other studies. ${ }^{6,12}$ Fever occurred in $17.39 \%$ of cases in present study and similar finding were not reported from Shrestha BM10 (7.4\%). Previous studies showed that headache and vomiting occurred due to destruction of cysts by antihelmintic drugs if given to a child with severe cerebral edema as it may precipitate further swelling and edema6. Prophylactic dexamethasone is beneficial in decreasing the severity of such symptoms. ${ }^{6}$

Both caste and ethnicity have a significant impact on the health and diseases of children. The concept of health and illness, their lifestyles, values and normature system may affect the contraction of diseases. In this study, Brahmin and Chhetri are the two major ethnic groups suffering from NCC the most. It is hard to explain. However, contamination of human soil in drinking water/vegetable might have contributed. ${ }^{14}$

Clinical symptoms at presentation like up rolling of both eyes present in significant percentage of children (26\%.), stiffening of the limbs in $17.39 \%$ and loss of consciousness present in $13 \%$ of cases in this study and such findings are not much mentioned previous studies.

\section{CONCLUSION}

Present study suggests that CT scanning of head or MRI is mandatory in all cases with seizure after the ages of five. Personal hygiene, water sanitation, sewage disposal must be improved. Use of human soil as a fertilizer in the field and garden must be discouraged. Such study needs to be carried out in larger scale in future.

\section{REFERENCES}

1. Dhawan B K edit. Pediatric Neurocysticercosis: Russell W Steel MD; (Available in http//emed.medscap.com/article 99053 overview.

2. Jnpp.bmj.com 2011. Neurocysticercosis and epilepsy in developing countries.

3. Singhi P, Singhi S. Neurocysticercosis in children. Indian journal of pediatrics 2009; vol. 76.

4. Kliegman; Nelson Text book of pediatrics 18 edition.

5. Karla V. A profile of childhood Neurocysticercosis. Indian Journal of Pediatrics 1994; 61:33-42.

6. Kossof EH. Neurocysticercosis. 2003;www.emedicine. com/ped/topic $1573 \mathrm{htm}$.

7. Chatterjee KD. Parasitology 2009; 13th edition.

8. Singhi P, Raj M, Singhi S et al. Clinical spectrum of 500 children with neurocysticercosis and response to albendazole therapy. Indian journal of neurology 2000 ;15:207-13.

9. Klara V, Sethi A. Childhood Neurocysticercosis; Epidemiology, Diagnosis and Course: Acta Pediatrica Japonica: 1992; 37:365-70.

10. Shrestha BM. Childhood Neurocysticercosis; Clinicoradiological profile and outcome.

11. Neurocysticercosis in a preschool age child; a case report; Arquinos de neuro-paquichia 2002;58:902-12.

12. Thakur LC, Ananda KS. Childhood Neurocysticercosis in South India. Indian Journal of Pediatrics 1991; 58:15-19.

13. Moralis NM, Agapejev S, Moralis RR et al. Clinical Aspects of Neurocysticercosis in Children; Pediatric Neurology. 2000;22: 287-91. 